\title{
Implantação do laboratório de simulação clínica de uma escola médica no interior do nordeste brasileiro: reflexões sobre o processo
}

Hugo Wesley de Araújo ${ }^{1}$ (D), Emanuelly Gomes Dário Santos ${ }^{1}$ (D), Gabriel Soares Dutra de Souza ${ }^{1}$ (D), Vivianne Izabelle de Araújo Baptista ${ }^{1}$ (D), Bruno Oliveira Carreiro ${ }^{1}$ (D), Raphael Raniere de Oliveira Costa $^{1}$ (D)

\begin{abstract}
RESUMO
Esse relato de experiência teve por objetivo descrever e refletir sobre o processo de implantação da simulação no Laboratório de Habilidades Clínicas e Simulação da Escola Multicampi de Ciências Médicas do Rio Grande do Norte (EMCM), bem como conjecturar sobre suas contribuições para a graduação de Medicina. No relato, foram resgatadas as vivências de estudantes e docentes da EMCM, descreve-se os cenários de prática, os recursos humanos, os simuladores, o público-alvo, as práticas simuladas, e as perspectivas para o futuro. Espera-se que a implantação do Laboratório de Habilidades Clínicas e Simulação possa fortalecer o processo de interiorização do ensino da Medicina e o compromisso da EMCM no cenário da Educação Médica, formando médicos com capacidade para transformar a realidade local, melhorando os indicadores de saúde e exercendo a profissão com destreza e dedicação.
\end{abstract}

Palavras-chave: Educação médica, Treinamento por simulação, Competência clínica.

\section{INTRODUÇÃO}

A Simulação Clínica é um método de aprendizagem ativa que possibilita a criação de cenários reais, em ambientes controlados, para a prática de habilidades e desenvolvimento de competências. Essa estratégia pode ser classificada em níveis de complexidade, a depender do grau tecnológico dos simuladores utilizados, dos objetivos e propósitos da atividade, do nível de experiência dos participantes e do conteúdo a ser abordado nos cenários ${ }^{1}$.

No contexto da Educação Médica, a simulação clínica pode ser empregada em diferentes momentos da formação e em diferentes contextos e cenários de práticas ${ }^{2}$. Em todas as realidades, os discentes vivenciam problemas reais, inclusive os que dificilmente são oportunizados em cenários de prática, e podem refletir sobre seus desempenhos, de modo a identificar as necessidades de aprendizagem em ambiente controlado ${ }^{3}$.

Durante as experiências clínicas simuladas, os cenários fazem referência a situações vivenciadas, por exemplo, em contexto hospita- lar ou de Atenção Básica, onde são trabalhadas habilidades cognitivas, psicomotoras, atitudinais e de comunicação. Dessa forma, o estudante desenvolve competências e habilidades em ambiente seguro, minimizando as possibilidades de erros na prática médica ${ }^{4,5}$. Além disso, aumenta a satisfação, autoeficácia, e autoconfiança na aprendizagem ${ }^{6}$.

Diante disso e das evidências de alta prevalência de erros médicos registrados pelo Committee on Quality in Health Care in America7, as Diretrizes Curriculares Nacionais para cursos de graduação em Medicina no Brasil determinam que o estudante de medicina deve "aprender em situações e ambientes protegidos e controlados, ou em simulações da realidade, identificando e avaliando o erro"8 (p.3). Assim, os cursos de Medicina passaram a ter a obrigatoriedade de oferecer ambientes de aprendizagem baseados em Simulação Clínica, fator que norteou a introdução desse método no projeto pedagógico da Escola Multicampi de Ciências Médicas do Rio Grande do Norte (EMCM) da Universidade Federal do Rio Grande do Norte?.

${ }^{1}$ Universidade Federal do Rio Grande do Norte. Curso de Medicina. Escola Multicampi de Ciências Médicas, Caicó (RN), Brasil. 
A EMCM é uma instituição pública de ensino superior, localizada no interior do estado do Rio Grande do Norte, que utiliza abordagens de ensino centradas no aluno através de Metodologias Ativas (MA), a exemplo da aprendizagem baseada em problema (ABP). O currículo obrigatório é dividido em dois eixos principais: o eixo tutorial e o eixo habilidades e comunidade, estando as práticas de simulação inseridas nesse último eixo. Essas atividades ocorrem no Laboratório de Habilidades Clínicas e Simulação, sendo este espaço um elemento fundamental para a efetivação e manutenção dos propósitos da Escola.

Laboratórios de Habilidades Clínicas e Simulação (LHCS) da EMCM tem por objetivo propiciar aos estudantes as condições necessárias para articular teoria e prática e fomentar o desenvolvimento de competências e habilidades gerais e específicas pertinentes a formação médica. Atualmente, conta com uma infraestrutura de ponta, constituída por um arsenal significativo de simuladores de baixa, média, alta fidelidade e simuladores de realidade virtual.

Destaca-se o protagonismo da EMCM no que diz respeito a interiorização do ensino da Medicina e da Simulação Clínica no Brasil. A simulação, antes vista somente nos grandes centros universitários e em países desenvolvidos, agora passa a fazer parte dos cenários de práticas das regiões mais remotas do Brasil e escassas de recursos tecnológicos para o ensino médico, possibilitando uma formação de qualidade. Dessa forma, este trabalho tem por objetivo descrever e refletir sobre o processo de implantação da simulação no Laboratório de Habilidades Clínicas e Simulação da Escola Multicampi de Ciências Médicas do Rio Grande do Norte, bem como conjecturar sobre suas contribuições para a graduação de Medicina.

\section{METODOLOGIA}

Trata-se de um relato de experiência realizado a partir do processo de implantação da simulação no LHCS da EMCM. Foram descritos os cenários de prática, os recursos humanos, os si- muladores, o público-alvo, as práticas simuladas, e as perspectivas para o futuro.

As reflexões foram tecidas concomitantemente a um levantamento teórico e documental da Resolução $n .{ }^{\circ} 1$ de fevereiro de $2019^{10}$, que dispõe sobre as normas de utilização do Laboratório, e o catálogo de simuladores da instituição $0^{11}$. Somado a isto, as experiências dos estudantes e docentes nas práticas desenvolvidas no laboratório.

\section{RESULTADOS E DISCUSSÃO}

\section{O cenário de práticas}

O LHCS é composto por oito espaços: uma sala de almoxarifado, um espaço para o treino de habilidades contendo área para o trabalho coletivo e três consultórios, duas salas de simulação avançada e uma sala de controle. Todas as salas foram projetadas para comportar as necessidades de flexibilidade, ambientação e gerenciamento de fluxos que podem surgir na criação dos diferentes cenários de simulação.

É válido destacar que os ambientes do laboratório não possuem estrutura fixa, exceto o almoxarifado, que serve para armazenamento de simuladores e insumos. Assim, para a ambientação dos cenários de assistência à saúde, a equipe do laboratório realiza as adequações do mobiliário e montagens necessárias. Como em grandes centros, é de fundamental relevância a possibilidade de criar e recriar espaços para os cenários planejados pelos docentes. Portanto, a mobilidade e flexibilidade são aspectos imprescindíveis ao processo de ambientação.

A sala de controle está localizada entre as duas salas de simulação avançada, e recebe esse nome, pois possui dois vidros espelhados com visão unidirecional que possibilita ao docente interagir com as salas de simulação, controlando os desfechos dos cenários durante a aplicação. A comunicação entre esses espaços ocorre através de um sistema de comando vocal, à distância, dos simuladores de alta fidelidade. A Figura 1 apresenta um desenho representativo da estrutura atual do LHCS. 
Figura 1 - Estrutura do Laboratório de Habilidades Clínicas e Simulação da Escola Multicampi de Ciências Médicas do Rio Grande do Norte. Caicó, 2020.

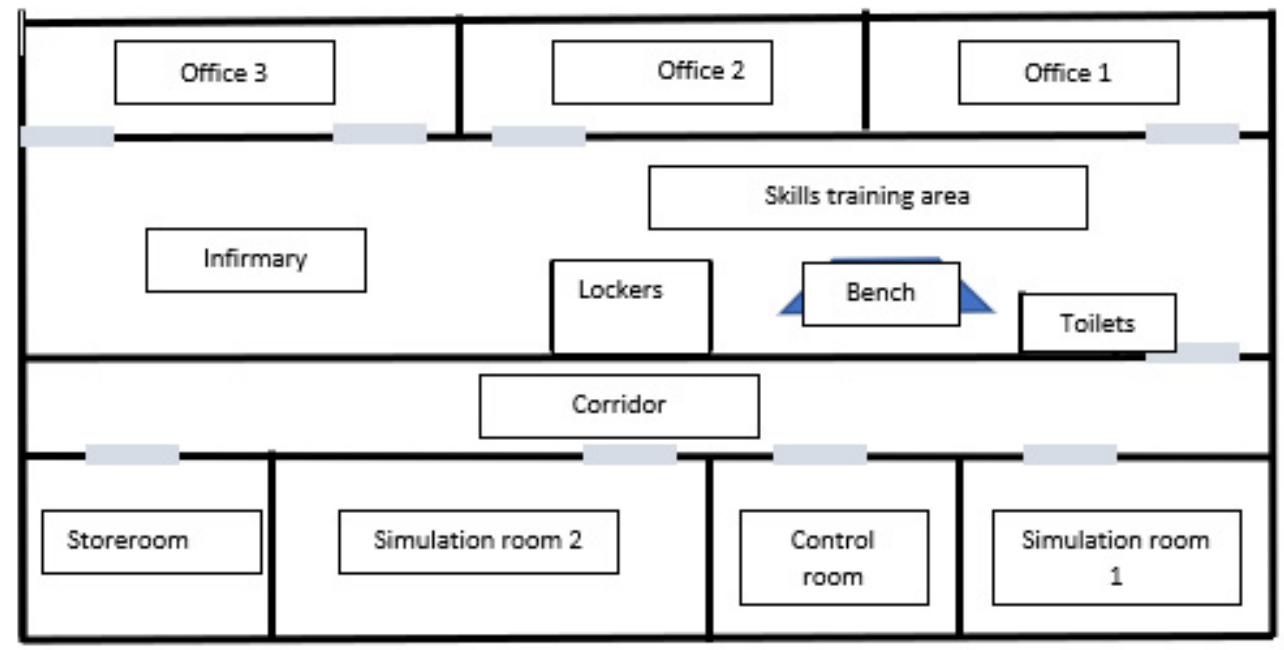

Os ambientes do LHCS ainda não possuem sistema próprio de captação e gravação de áudio e vídeo. Nesse sentido, há dificuldades de gravação dos cenários e do uso das imagens de vídeo para a discussão dos casos que foram simulados. Entretanto, a equipe de Tecnologia da Informação da EMCM fornece suporte para a captação desses recursos quando necessário.

Além disso, há uma iniciativa dos estudantes do Módulo de Inovação Tecnológica em Saúde para o desenvolvimento de um sistema audiovisual de baixo custo, uma vez que a utilização de recursos dessa natureza favorece a realização do debriefing, o componente mais importante de qualquer simulação ${ }^{12}$, e é recomendada pela Internacional Nursing Association for Clinical Simulation and Learning (INACSL) ${ }^{13}$, órgão internacional de referência na área de simulação clínica. Nessa perspectiva, os recursos audiovisuais podem contribuir para a visualização de atitudes e posturas que o aprendiz assume durante o cenário.

É importante destacar que, embora recomendada pela INACSL, este recurso é opcional, sobretudo para laboratórios que trabalham somente com estudantes de graduação. Além disso, a sua indisponibilidade não inviabiliza as sessões de simulação. Na realidade em relato, os docentes apontam para a necessidade de tê-lo com o objetivo de filmar cenários em provas práticas, na residência médica, e até mesmo utilizar este recurso em capacitações de profissionais da rede de atenção à saúde local.

O debriefing é uma sessão estruturada que ocorre após a realização do cenário, com o objetivo de potencializar a experiência vivida e potencializar o significado do que foi experimentado $^{3,14,15,3}$. Na EMCM, os docentes são estimulados a fazer uso do debriefing estruturado por sua natureza interativa, reflexiva e motivadora que permite desenvolver o pensamento estruturado.

\section{Recursos humanos}

A escola dispõe de profissionais técnico-administrativos alocados para apoio aos docentes na montagem dos cenários e equipamentos. Esses profissionais também são responsáveis por fornecer suporte a monitores e estudantes nas aulas práticas, garantindo o manuseio adequado dos simuladores. Além disso, diante da expansão do laboratório, das práticas simuladas e do fortalecimento da simulação no currículo do curso, no ano de 2018 foi realizado um concurso docente para contratação de profissionais com experiência na área de simulação clínica.

As capacitações dos técnicos do laboratório, sobretudo no manuseio de simuladores, principal- 
mente os de alta fidelidade, são realizadas pelas empresas responsáveis pela fabricação e/ou venda do produto. Para os professores que não foram treinados no manuseio das tecnologias, e que necessitam utilizar os simuladores, é responsabilidade dos técnicos treiná-los. Assim, garante-se maior durabilidade dos materiais, bem como o uso ampliado dos recursos do laboratório.

A capacitação docente - no que se refere à estratégia da simulação enquanto ferramenta pedagógica - também tem sido viabilizada com o objetivo de melhorar, do ponto de vista metodológico e pedagógico, as práticas simuladas. Na EMCM, as experiências clínicas simuladas se fazem presentes em todos os módulos do curso de Medicina. Logo, essa agenda de capacitação é cumprida por docentes especialistas e é de suma relevância para o aperfeiçoamento da estratégia.

Sabe-se que os simuladores são recursos e que, por si só, não garantem que a simulação atinja os objetivos de aprendizagem previstos no plano de curso e no currículo. Nessa perspectiva, é importante ter a clareza de que além de dispor de diferentes recursos como os simuladores, é indispensável promover capacitações para o corpo docente.

\section{Os simuladores}

Os simuladores são classificados de acordo com seu grau de fidelidade: baixa, média e alta. Os simuladores de baixa fidelidade são modelos ou manequins usados para reconhecimento de estruturas e prática de procedimentos simples. Os de média fidelidade possuem capacidade limitada de interação usuário-máquina, enquanto os de alta fidelidade são comandados por computadores e geram diferentes respostas a depender da realização ou não da tarefa pelos usuários ${ }^{16}$. Além disso, ainda há os simuladores de Realidade Virtual (RV). Atualmente, a Escola dispõe de sessenta modelos de simuladores, distribuídos nos diferentes níveis de fidelidade, a saber: baixa, média, e alta fidelidades; bem como simuladores de realidade virtual.

Somando-se aos simuladores, é importante destacar a relevância do paciente-padrão, também denominado paciente simulado. Estes são atores na função de paciente em Simulação de uma situação real' ${ }^{17}$. Há um consenso na literatura quanto ao uso de pacientes simulados para aquisição de variados ganhos, como autoconfiança, capacidade para resolução de conflitos, melhoria do trabalho em equipe, de habilidades técnicas e de comunicação ${ }^{18,19,20}$, maior satisfação, empatia, realismo, menor nível de ansiedade, maior incentivo a capacidade de reflexão e pensamento crítico ${ }^{17}$.

$\mathrm{Na}$ EMCM, não há banco de atores. Porém, os projetos de monitoria vinculados aos módulos do curso preveem a participação dos monitores nas atividades que necessitam do recurso de dramatização e paciente simulado.

\section{Público-alvo}

O laboratório e os equipamentos de simulação da EMCM são destinados ao desenvolvimento de habilidades psicomotoras, atitudinais e de comunicação dos estudantes da graduação de Medicina, das Residências Médicas em Clínica Médica, Cirurgia Geral e Medicina de Família e Comunidade. Além disso, pode atender às demandas da Residência Multiprofissional em Atenção Básica, da Residência Multiprofissional Materno-Infantil, e do Programa de Pós-Graduação em Educação, Trabalho e Inovação em Medicina (Mestrado Profissional).

Além das atividades regulares promovidas pelos docentes, os estudantes e monitores possuem acesso ao laboratório para treinamento das práticas aprendidas durante as aulas de simulação/habilidades. A circulação dos alunos é controlada pelos técnicos de laboratório que os auxiliam no manuseio dos equipamentos. Há também um regimento interno e os fluxos para agendamento das práticas e solicitação de materiais/simuladores ${ }^{10}$.

O processo de desenvolvimento de competências e habilidades dentro do laboratório acompanha os estudantes de graduação durante toda a sua formação, tendo um espaço semanal reservado para desenvolvimento dessas destrezas: do primeiro semestre ao internato, da residência ao mestrado. 


\section{As práticas simuladas na EMCM}

Para utilização dos espaços do laboratório pelos docentes, deve-se realizar o agendamento eletrônico da atividade. Além disso, os responsáveis pela simulação realizam a construção de um roteiro de habilidades que contém: identificação da atividade; objetivos de aprendizagem; os recursos humanos, físicos e materiais necessários; descrição detalhada do que será realizado e referências bibliográficas.

As práticas de habilidade variam em graus de fidelidade e complexidade. Algumas simulam apenas a realização de procedimentos, buscando aperfeiçoar o estudante no passo-a-passo de técnicas, enquanto outras simulam, além de abordagens técnicas, a ambientação e comunicação com o paciente.

As simulações são organizadas de acordo com os módulos de ensino desde o primeiro semestre de aulas. Como cada módulo apresenta objetivos diferentes, as simulações correspondem às competências e habilidades determinadas em cada um desses componentes. Assim, os alunos participam de cenários de diferentes níveis de complexidade e fidelidade.

Além dos simuladores, a escola tem investido em técnicas de moulage. A moulage é um recurso que envolve técnicas de modelagem e maquiagem em modelo vivo. No contexto da EMCM, uma das principais aplicações tem sido representar fielmente tecidos de ferimentos de pele. Assim, é utilizada nos módulos de Urgência e Emergência, em que aborda pacientes politraumatizados e queimados, e em aulas sobre manejo de feridas de pele na Atenção Primária à Saúde e no internamento hospitalar.

A Escola também tem apostado, para aulas e avaliações, em estações simuladas que integram conhecimentos das Ciências Básicas e a Clínica. Por exemplo, após a coleta do exame preventivo de colo de útero, os alunos são desafiados a identificar os aspectos citológicos e microbiológicos daquela coleta em lâmina de microscópio, dessa forma, em uma mesma estação são trabalhados conteúdo da Semiologia, Ginecologia, Anatomia e Microbiologia. 0 objetivo dessas práticas é integrar o conhecimento.

As avaliações de habilidades são realizadas através do Objective, Structured Clinical Exami- nation (OSCE) e Clinical Skills Assessment (CSA). $\mathrm{Na}$ maioria das estações, os discentes entram individualmente, recebem um comando contendo informações fictícias relacionadas ao ambiente de trabalho, ao paciente e a tarefa que devem executar, por exemplo, a realização de um procedimento, o exame clínico ou manejo terapêutico.

São construídas fichas de avaliação contendo os pontos a serem pontuados no desempenho dos discentes ao longo da atividade. Nessas fichas, a depender do grau de fidelidade, o aluno pode ser avaliado de acordo com sua relação com o paciente, com o seu desempenho técnico em realizar determinado procedimento ou entrevista clínica e se sua conduta foi resolutiva para o problema apresentado.

Há também uma iniciativa no sentido de usar a simulação clínica como disparador na abertura de problemas no método ABP. Em módulos mais avançados, os estudantes participam de um cenário simulado, em que, por exemplo, podem atender um paciente e, posteriormente, retornarem ao grupo tutorial para dar seguimento aos passos do método ABP. A experiência tem sido bastante positiva e bem avaliada entre os estudantes.

\section{Perspectiva para o futuro e os desafios}

Atualmente, há uma tendência quanto ao uso da simulação clínica e de simuladores no contexto da formação médica. Entretanto, é válido considerar que esse método é apenas uma das possibilidades que o docente pode fazer uso. A depender dos objetivos de aprendizagem, outras estratégias podem ser utilizadas durante o processo de ensino e aprendizagem.

Ao fazer a opção pela simulação e pelo uso de simuladores, o docente precisa ter conhecimento das etapas da estratégia, e ter uma postura compatível ao que se espera de um tutor que faz uso de MA. Para tanto, a capacitação docente parece ser indispensável. $O$ investimento em simuladores também se faz necessário.

A simulação de situações-problema permite que o estudante possa aprender com seus próprios erros e acertos sem que isso implique em iatrogenia a um paciente real, dessa forma, ele estará mais capacitado para lidar com essas 
situações em um cenário real, tendo uma maior chance de resolução da necessidade de saúde do usuário ${ }^{21,5,22}$. Fazer uso da simulação também é uma escolha acertada do ponto de vista da igualdade. Na EMCM, pelo seu caráter multicampi, os estudantes participam de atividades em diferentes rodízios, serviços de saúde e cidades. Com isso, não se pode garantir que todos tenham acesso igualitários aos casos vivenciados durante as práticas clínicas nesses serviços. Nesse sentido, a simulação pode preencher a lacuna da igualdade de acesso ao treinamento de habilidades indispensáveis a formação médica ${ }^{22}$.

No contexto relatado, aposta-se também nas simulações de alta fidelidade e complexidade. Essas características são especialmente valiosas já que o estudante é inserido na comunidade desde o primeiro período do curso. Sendo assim, o contato com simulações torna-se uma ferramenta para o aprimoramento das habilidades que serão utilizadas e requisitadas ao longo do processo de formação médica ${ }^{23,24}$.

Todas essas oportunidades são frutos da interiorização do ensino da Medicina e do investimento na estruturação do Centro de Simulação. Atualmente, entre as universidades públicas federais, a EMCM ocupa um lugar privilegiado quando se fala em simulação e infraestrutura para as práticas simuladas.

Para o futuro, espera-se que o novo centro de simulação, em construção, possa atender as demandas e a crescente utilização da simulação pelos docentes da Escola. Pretende-se também buscar o título de acreditação fornecido pelas entidades latino-americanas de Simulação Clínica para o Centro de Simulação da EMCM. Na Figura 2 , apresenta-se um desenho representativo do novo centro de simulação.

Figura 2 - Estrutura do Laboratório de Habilidades Clínicas e Simulação da Escola Multicampi de Ciências Médicas do Rio Grande do Norte, em construção. Caicó, 2020.

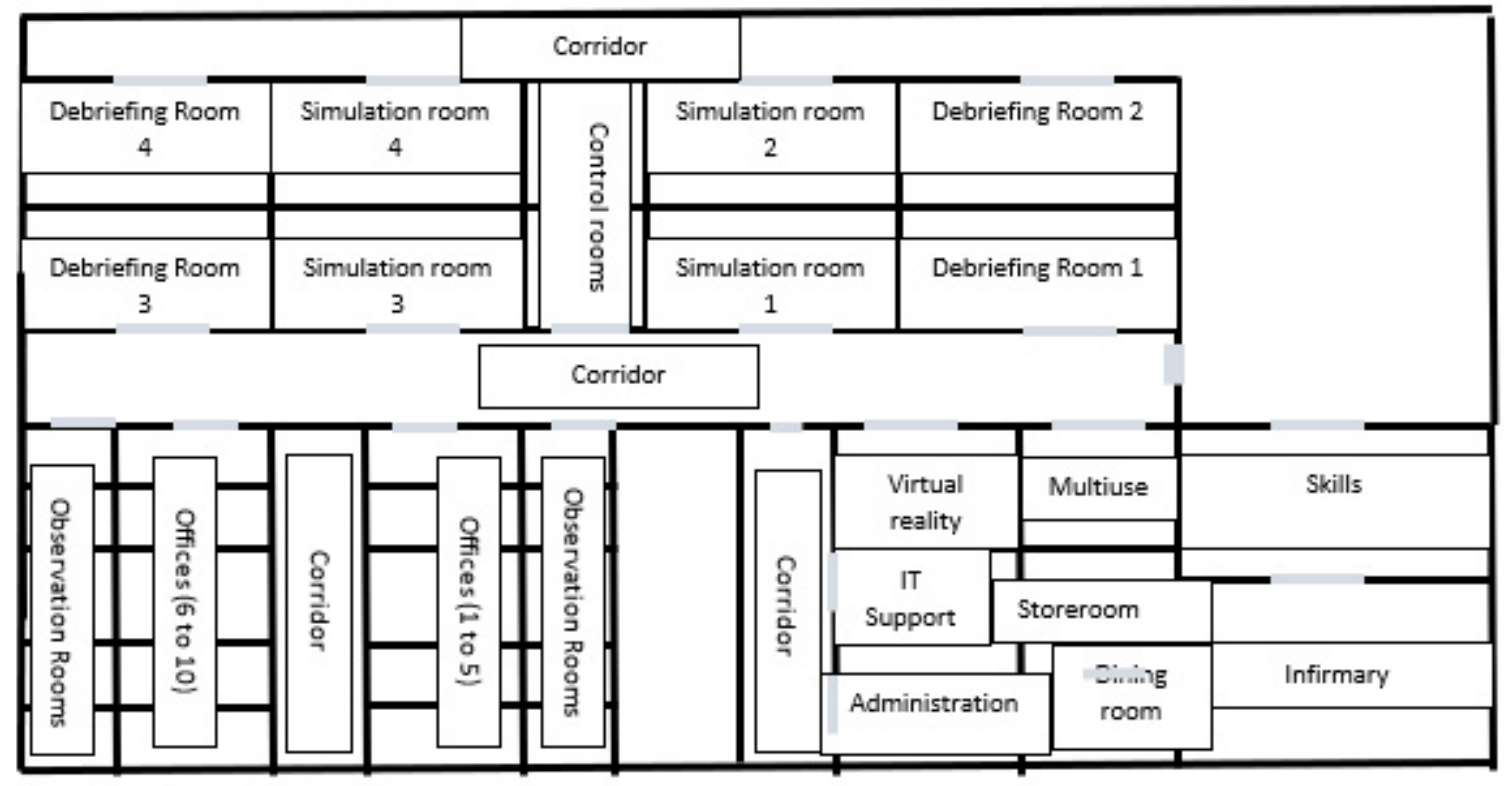


Além disso, espera-se que as práticas simuladas possam se fazer presentes em diferentes momentos dos módulos do Curso de Medicina da EMCM e do internato. Também serão buscados investimentos para aquisição de simuladores de realidade virtual e a criação do banco de atores.

Somando a isto, o desenvolvimento de investigações para que se possa mensurar, de modo mais concreto, o impacto do uso da simulação no contexto formativo objeto desse relato. A qualificação contínua do corpo docente, a aquisição e reposição de simuladores e outras tecnologias, a divulgação de práticas bem-sucedidas, bem como a busca por editais de financiamento, também são objetivos para o futuro.

\section{CONSIDERAÇÕES FINAIS}

A simulação clínica tem ganhado espaço diante da valorização de currículos que respondam à necessidade crescente de abordagens integrais em saúde e que possibilitem a aproximação entre a teoria e a prática profissional. A construção de cenários de prática protegidos e controlados permite que $o$ indivíduo pratique exaustivamente, aprenda, reflita e avalie produtos e processos de seu desempenho, resultando na aquisição de competências importantes para as profissões em saúde. Tudo de forma ética e segura, proporcionando, ainda, a homogeneização das oportunidades de aprendizado.

Não à toa, a construção de espaços de simulação foi valorizada pelas Diretrizes Curriculares Nacionais de Medicina ${ }^{10}$ corroborando com as associações realizadas pela literatura que a apontam como importante ferramenta pedagógica no desenvolvimento de habilidades técnicas e de comunicação, desenvolvimento da capacidade de resolução de problemas e do raciocínio interdisciplinar.

Na EMCM, a prática da simulação tem papel primordial no processo de ensino-aprendizagem pautado no protagonismo do aluno e na ressignificação da prática médica de excelência, sendo uma das metodologias de escolha para o ensino do eixo habilidades. A Escola tem assumido um papel de liderança, tanto em quantitativo quanto qualitativo, entre as universidades públicas fede- rais brasileiras no que diz respeito a simulação e suas tecnologias.

Espera-se que a implantação do Laboratório de Habilidades Clínicas e Simulação da EMCM possa fortalecer o processo de interiorização do ensino da medicina e o compromisso da EMCM no cenário da educação médica. Espera-se que os futuros médicos possam transformar as realidades que venham a ser inseridos, melhorando indicadores de saúde e exercendo a profissão com destreza e dedicação.

\section{REFERÊNCIAS}

So HY, Chen PP, Kwok G, Wong C, Tung T, Chan N. Simulation in medical education. J R Coll Physicians Edinb. 2019;49(1):52-7.

Moura-Júnior LG de, Ramos A, Campos JM, Ferraz ÁA, Rocha HÂL, Costa GO. Teaching model for evaluation of the ability and competence progress in endosuture in surgical skill laboratory. Arquivos brasileiros de cirurgia digestiva: $A B C D=$ Brazilian archives of digestive surgery. 2017 Oct 1;30(4):256-9.

Martins J. Learning and development in simulated practice environments. Revista de Enfermagem Referência. 2017; IV Série(12):155-62.

Almeida RG dos S, Mazzo A, Martins JCA, Baptista RCN, Girão FB, Mendes IAC. Validation to Portuguese of the scale of student satisfaction and self-confidence in learning. Revista Latino-Americana de Enfermagem. 2015 Nov $1 ; 23(6): 1007-13$.

Varga CRR, Almeida VC, Germano CMR, Melo DG, Chachá SGF, Souto BGA, Fontanella, BJB, Lima VV. Report on an experience with simulations in the teaching: learning process in medicine Mestrado em Gestão da Clínica View project Molecular Basis of Ablepharon Macrostomia Syndrome View project. Revista Brasileira de Educação Médica [Internet]. 2009 [cited 2019 Dec 10];33(2):291-7. Available from: https://www.researchgate.net/publication/262748520

Costa RRO, MEDEIROS SM, Martins JCA, Coutinho VRD. Simulation in training nurses: reflections and justifications based on bioethics and human rights approaches. Acta Bioethica, 2018; 24(1): 31-38.

Committee on Quality in Health Care in America, Institute of Medicine. Crossing the Quality Chasm. A New Health System for the 21st Century. Washington DC: National Academy Press; 2000.

Committee on Quality in Health Care in America. Institute of Medicine. Crossing the Quality Chasm: A New Health System for the 21st Century. National Academy Press. 2001. 360.

Ministério da Educação (BR). Conselho Nacional de Educação. Câmara de Educação Superior. Resolução CNE/CES 
$n^{\circ}$ 3, de 20 de junho de 2014. Institui as Diretrizes Nacionais do Curso de graduação em Medicina. Diário Oficial da União. 23 jun 2014.

Universidade Federal do Rio Grande do Norte. Projeto Pedagógico do Curso de Medicina CERES-FACISA/UFRN. 2014 p. 101.

Resolução Nº 01 de 05 de fevereiro de 2019. Dispõe sobre as normas de utilização dos Laboratórios de Habilidades Clínicas e Simulação da Escola Multicampi de Ciências Médicas do Rio Grande do Norte. 2019 p. 4.

Costa RRO, Medeiros SM, Martins JCA, Dias VR. Percepções de estudantes de enfermagem acerca das dimensões estruturais da simulação clínica. Sci Med. 2019;29(1):e32972.

Luna-Villanueva E, Santos-Rodríguez M de los, Sierra Basto G, González-Arriaga CR, Zamora-Graniel FG. Retroalimentación integral (debriefing) oral y asistida por video en simulación de reanimación cardiopulmonar avanzada: estudio piloto. FEM: Revista de la Fundación Educación Médica [Internet]. 2015 [cited 2019 Dec $10] ; 18(2): 139-47$. Available from: www.fundacioneducacionmedica.org

Standards Committee I. INACSL Standards of Best Practice: Simulation SM Debriefing. Clinical Simulation in Nursing [Internet]. 2016 [cited 2019 Dec 10];12:S21-5. Available from: http://dx.doi.org/10.1016/j.ecns.2016.09.008

Kolbe M, Grande B, Spahn DR. Briefing and debriefing during simulation-based training and beyond: Content, structure, attitude and setting [Internet]. Vol. 29, Best Practice and Research: Clinical Anaesthesiology. 2015 [cited 2019 Dec 10]. p. 87-96. Available from: https://www.sciencedirect.com/science/article/pii/S1521689615000038

Gardner R. Introduction to debriefing [Internet]. Vol. 37, Seminars in Perinatology. 2013 [cited 2019 Dec 10]. p. 166-74. Available from: https://www.sciencedirect. com/science/article/pii/S014600051300030X

Martins JCA, Mazzo A, Baptista RCN, Coutinho VRD, de Godoy S, Mendes IAC, Trevizan MA. A experiência clínica simulada no ensino de enfermagem: retrospectiva histórica. Acta Paul Enferm. 2012. 25(4):619-25.

Negri EC, Mazzo A, Martins JCA, Pereira Junior GA, Almeida RG dos S, Pedersoli CE. Simulação clínica com dramatização: Ganhos percebidos por estudantes e profissionais de saúde. Vol. 25, Revista Latino-Americana de Enfermagem. University of Sao Paulo, Ribeirao Preto College of Nursing Organisation; 2017.

Pagano MP. Health Communication for Health Care Professionals [Internet]. Springer Publishing Company. New York: Springer Publishing Company; 2016 [cited 2019 Dec 10]. Available from: https://books.google.com.br/books?hl=p$\mathrm{t}-\mathrm{BR} \& \mid \mathrm{r}=\& \mathrm{id}=\mathrm{gCYODAAAQBAJ} \& \mathrm{i}=\mathrm{fnd} \& \mathrm{pg}=\mathrm{PP} 1 \& \mathrm{dq}=-$ Health+communication+for+health+care+professionals. + \&ots $=$ A1gQPjpvPC\&sig=k2C2 Bc9VMN4ILaHitcVrpztatA

Spinner-Gelfars AH. Using simulation to promote effective communication with a diverse student population. Teaching and Learning in Nursing. 2013 Jul;8(3):96-101.

Efstathiou N, Walker WM. Interprofessional, simulation-based training in end of life care communication: A pilot study. Journal of Interprofessional Care [Internet]. 2014 [cited 2019 Dec 10];28(1):68-70. Available from: http://informahealthcare.com/

Pazin Filho A, Scarpelini S. Simulação: Definição. In: Medicina. Faculdade de Medicina de Ribeirao Preto - U.S.P.; 2007. p. $162-6$

Costa RRO, Medeiros SM, Vitor AF, Lira ALBC, Martins JCA, Araujo MS. Types and purposes of the simulation in undergraduate nursing education: integrative literature review. Rev Baiana Enferm. [Internet]. 2016 Sep [cited Feb 22, 2018];30(3):1-11. doi: http://dx.doi. org/10.18471/rbe.v30i3.16589

e Oliveira $A L$ de $O$, de Melo LP, Pinto TR, de Azevedo GD, dos Santos M, da Câmara RBG, et al. Vivência integrada na comunidade: inserção longitudinal no Sistema de Saúde como estratégia de formação médica. Interface: Communication, Health, Education [Internet]. 2017 [cited 2019 Dec 10];21(Supp 1):1355-65. Available from: https://www.scielosp.org/article/icse/2017.v21suppl1/1355-1366/

de Melo LP, dos Santos M, da Câmara RBG, Braga LP, E Oliveira AL de $O$, Pinto TR, et al. A escola multicampi de ciências médicas da universidade federal do Rio Grande do norte, Brasil, no contexto do programa mais Médicos: Desafios e potencialidades. Interface: Communication, Health, Education [Internet]. 2017 [cited 2019 Dec 10];21(Suppl 1):1333-43. Available from: https://www.scielosp. org/article/icse/2017.v21suppl1/1333-1343/es/ 


\section{CONFLITO DE INTERESSES}

Não existem conflitos de interesses. Nenhum financiamento foi fornecido para este estudo.

Autor correspondente:

Raphael Raniere de Oliveira Costa

raphaelraniere@hotmail.com

Editor:

Prof. Dr Felipe Villela Gomes

Recebido: $27 / 07 / 2020$

Aprovado: $16 / 09 / 2020$

c) (i) Este é um artigo publicado em acesso aberto (Open Access) sob a licença Creative 